\title{
Entanglement enhancement for two spins assisted by two phase kicks
}

\author{
Gennadiy Burlak ${ }^{1}$, Isabel Sainz ${ }^{2}$, and Andrei B. Klimov ${ }^{2 *}$ \\ ${ }^{1}$ Centro de Investigación en Ingeniería y Ciencias Aplicadas, \\ Universidad Autónoma del Estado de Morelos, Cuernavaca, Morelos, México \\ ${ }^{2}$ Departamento de Física, Universidad de Guadalajara, \\ Revolución 1500, Guadalajara, Jalisco, 44420, México.
}

\begin{abstract}
We study the entanglement dynamics in a two-spin system governed by a bilinear Hamiltonian and assisted by phase kicks. It is found that the application of instant kicks to both spins at some specific moments leads to enhancement of entanglement. This procedure also improves the transient character of entanglement leading, for large spins, to a formation of a plateau for the I-concurrence. We have numerically investigated the spin-spin dynamics for several values of spins and observed a substantial enhancement of entanglement in comparison to the evolution without kicks.
\end{abstract}

\footnotetext{
*klimov@cencar.udg.mx
} 


\section{INTRODUCTION}

Recently, a significant amount of theoretical and experimental research has been carried out for generating, manipulating and preserving entanglement, which is the key ingredient for developing quantum technologies. The main attention is usually paid to studying entanglement properties of qubits (two-level systems) (see e.g. [1, 2]), considered as a basic physical resource. Although, higher dimensional systems (qudits) could be more appropriate for certain physical applications, entanglement dynamics, generation and manipulation in higher dimensions is not as well studied as in two-dimensions.

In particular, it was suggested in [3] that it is possible to increase the security, the bit transmission rate, or both in quantum key distribution protocols by increasing the dimensionality of the involved systems. The possibility to perform quantum computation based on qudits cluster states [4] (has also been noted). Nevertheless, just few years ago some studies on entanglement dynamics in higher dimensions have been done. For example, the dynamics of a initially entangled two-qudit state was studied in [5], while in [6] it is shown that two non-interacting, initially separable spins can get entangled via a common purely dephasing environment. More general results about environment mediated generation of entanglement in higher dimensions can be found in [7]. Other aspects of entanglement in higher dimensional systems, like entanglement concentration for two qudits in [8], and its experimental implementation for two qutrits [9] were reported. It was also shown in [10] that the quality of entanglement transfer in spin chains actually increases with the dimension of the spin.

On the other hand, higher dimensional systems (real, as high excited nuclear spins or effective, as Dicke-like states) are of interest by themselves, especially in the context of studying the quantum-classical transition, when the system's dimension becomes sufficiently large. However, it is well-know that there are no reasons to expect that the classical behavior can be approached in the large dimension limit for every state [11]. In particular, the coherent spin states (CSS), $|\zeta, S\rangle,[12]$ are the most classical ones, and some specific linear combination of them may reveal non-classical features, like entanglement, even in the limit of large values of $S$ (see [13]).

One of the regular ways for generating and measuring entanglement in many spin $1 / 2$ system is through spin squeezing (see [14] and references therein), where quantum correla- 
tions naturally appear in the basic concept of squeezed spin states (SSS) [15]. The simplest Hamiltonian which produces squeezing starting with coherent states located on the equator

of the Bloch sphere is $H=g S_{z}^{2}$, where $S_{z}=\sum_{j} \sigma_{z}^{(j)} / 2$ is a collective spin operator [15].

Unfortunately, there are two problems with such dynamical generation of squeezing and entanglement: a) the squeezing (and entanglement) is always transient, i.e. it appears periodically ; b) it does not reach its maximal value, except the simplest two-qubit case.

In the case of large two spins the situation is rather similar. It is possible to generate in an easy way the entanglement between these spins, but it would resemble the above mentioned disadvantages as in the symmetric combination of many qubits.

In the present article we address the following question: is it possible to improve the entanglement properties of a two qudit system in a relatively simple way?

We will show that by applying instant kicks to both spins at some particular moments we can not only enhance the entanglement, but, more importantly, essentially improve its transient nature, i.e. reduce the distance between the maximum and the minimum values, so that the system evolves into an approximate "steady" state of the interaction Hamiltonian with a high value of entanglement for sufficiently large spins. We also discuss its possible application to the purely dephasing environment [6, 7].

\section{THE MODEL}

Let us consider two spins $S_{1}$ and $S_{2}$, of dimensions $2 S_{1}+1$ and $2 S_{2}+1$ respectively, which interact according to the Ising-like Hamiltonian,

$$
H=g \hat{S}_{z 1} \hat{S}_{z 2}
$$

This kind of interaction was proposed to implement one way quantum computation with many-level cluster states (see [4] and references therein). Suppose that each spin is initially in the eigenstate of $\hat{S}_{x i}$ with zero phase, $\hat{S}_{x i}\left|\zeta_{i}=1, S_{i}\right\rangle=S_{i}\left|\zeta_{i}=1, S_{i}\right\rangle$, which is a CSS placed on the equator of the Bloch sphere,

$$
\left|\zeta_{i}=1, S_{i}\right\rangle=\frac{1}{2^{S_{i}}} \sum_{k_{i}=-S_{i}}^{S_{i}} \sqrt{\frac{\left(2 S_{i}\right) !}{\left(S_{i}+k_{i}\right) !\left(S_{i}-k_{i}\right) !}}\left|k_{i}, S_{i}\right\rangle,
$$

where $i=1,2$ and $\hat{S}_{z i}\left|k_{i}, S_{i}\right\rangle=k_{i}\left|k_{i}, S_{i}\right\rangle$, so that the joined state for the system composed by spins $S_{1}$ and $S_{2}$ is $\left|\Psi_{0}\right\rangle=\left|\zeta_{1}=1, S_{1}\right\rangle\left|\zeta_{2}=1, S_{2}\right\rangle$. 
Since $\left|\Psi_{0}\right\rangle$ is not an eigenstate of the Hamiltonian, in the course of evolution the joined state

$$
|\Psi(t)\rangle=\hat{U}(t)\left|\Psi_{0}\right\rangle, \quad \hat{U}(t)=e^{-i t g \hat{S}_{z 1} \hat{S}_{z 2}}
$$

becomes entangled at some instants. As an entanglement measure we will use the (normalized) $I$-concurrence [16], that for pure states takes the form,

$$
\mathcal{C}_{I}=\frac{2 S+1}{2 S}\left(1-\operatorname{Tr} \hat{\rho}_{1(2)}^{2}\right)
$$

where $\hat{\rho}_{1(2)}=\operatorname{Tr}_{2(1)}(\hat{\rho}(t))$ is the density matrix of the composed system $\hat{\rho}(t)=|\Psi(t)\rangle\langle\Psi(t)|$ traced by the spin $S_{2}\left(S_{1}\right)$, and $S=\min \left(S_{1}, S_{2}\right)$, such that $0 \leq \mathcal{C}_{I} \leq 1$, being zero for separable states and one for maximally entangled states.

For the state (2), $\mathcal{C}_{I}=(2 S+1)(1-\mathcal{P}) / 2 S$, where the purity $\mathcal{P}=\operatorname{Tr} \hat{\rho}_{1}^{2}(t)$ is

$$
\mathcal{P}=\frac{1}{16^{S_{1}}} \sum_{k, l=-S_{1}}^{S_{1}} \frac{\left[\left(2 S_{1}\right) !\right]^{2} \cos ^{4 S_{2}}(g t(k-l) / 2)}{\left(S_{1}+k\right) !\left(S_{1}-k\right) !\left(S_{1}+l\right) !\left(S_{1}-l\right) !} .
$$

From now on we will focus only on the symmetric case: $S_{1}=S_{2}=S$. Let us consider the two simplest cases: $S=1 / 2,1$. For two qubits, $S=1 / 2, \mathcal{C}_{I}=\sin ^{2} g t / 2$, i.e. it oscillates between zero and one, so that the spins are maximally entangled at times $g t=(2 n+1) \pi$ and they are separable at times $g t=2 n \pi, n=0,1, \ldots$ Nevertheless, for $S=1$, the situation is already different, since $\mathcal{C}_{I}=3\left(4-2 \cos g t-\cos ^{2} g t-\cos ^{4} g t\right) / 8$, reaches its maximum value $\mathcal{C}_{I}^{\max } \approx 0.88$ at times $g t \approx 2.2,4.1+2 n \pi$, and thus the spins will never become maximally entangled, although they are disentangled at $g t=2 n \pi, n=0,1, \ldots$ The entanglement dynamics have a quasi-periodical behavior, as is shown by the (green) dash-dotted line in Fig. 1 (a).

In the large spin limit, $S \gg 1$, we can approximate the time average of $\mathcal{C}_{I}$ as follows,

$$
\left\langle\mathcal{C}_{I}\right\rangle \approx \frac{2 S+1}{2 S}\left(\frac{2}{\sqrt{2 \pi S}}-\frac{1}{2 \pi S}\right),
$$

giving $\left\langle\mathcal{C}_{I}\right\rangle \approx 0.54$ for $S=1$, which is quite close to the exact numerical value $\left\langle\mathcal{C}_{I}\right\rangle=0.58$, even if the approximation is done for $S \gg 1$.

\section{ENHANCING ENTANGLEMENT}

In order to enhance the maximum achievable entanglement let us apply instant pulses (kicks) to each spin at certain times $t_{k}$. Such kicks correspond to rotations around the $y$-axis, 


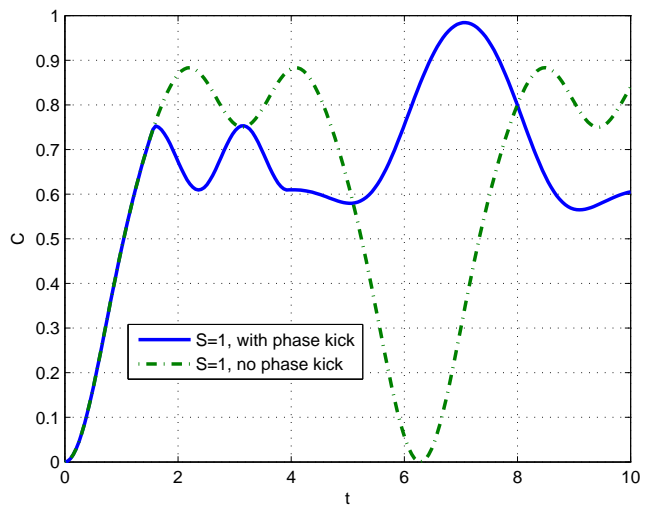

(a)

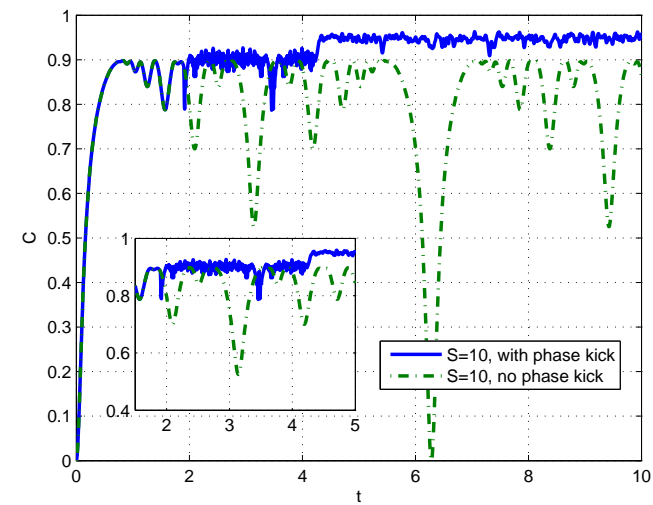

FIG. 1: (Color online) Time evolution of $\mathcal{C}_{I}$, without kicks (green, dash-dotted line) and with phase-kicks (blue, continuous line) for (a) $S=1$, with the numerical optimization times $t_{1}=1.6$ and $t_{2}=3.9$, and (b) $S=10$, in this case $t_{1}=1.9$ and $t_{2}=4.2$ where the inset shows the details for the second phase kick.

and for the $j$-th spin are represented by the operators $\hat{R}_{j}=e^{-i \pi \hat{S}_{y j} / 2}$. The main idea is as follows: initially both spins are in eigenstates of $\hat{S}_{x i}$, which can be represented by localized distributions on the equator of the corresponding Bloch spheres. In the course of evolution governed by Eq.(11) the state become partially entangled. At some appropriate moment $t_{1}$ we apply a $\pi / 2$-rotation to both spins so that each component of the angular momentum basis $\left|k_{i}, S\right\rangle$ instantly transforms into an eigenstate of $\hat{S}_{x i}$. Afterwards, the evolution under the Hamiltonian (11) is continued for a while, which continues coupling both spins. Then, at some time $t_{2}$ we apply the inverse $\pi / 2$-rotation that transforms again each element $\left|k_{i}, S\right\rangle$ into an eigenstate of $\hat{S}_{x i}$, after that we allow the system to evolve further:

$$
|\Psi(t)\rangle=\hat{U}\left(t-t_{2}\right) \hat{R}^{-1} \hat{U}\left(t_{2}-t_{1}\right) \hat{R} \hat{U}\left(t_{1}-t_{0}\right)\left|\Psi_{0}\right\rangle,
$$

where $\hat{R}=\hat{R}_{1} \otimes \hat{R}_{2}$. The instants $t_{1}$ and $t_{2}$ are chosen in order to optimize the maximum value of achievable entanglement after the second kick. A similar procedure was proposed to optimally create squeezing in Dicke states [17] and entanglement for continuous-variable systems in [18, 19].

The reason for applying $\pi / 2$-rotations can be explained as follows: we want to correlate as many components of the spin states as possible, which cannot be done only with the Hamiltonian evolution, since the number of components of the angular momentum basis, initially involved in the CSS, is of order $\sqrt{S}$. By applying the kicks at some specific moments 

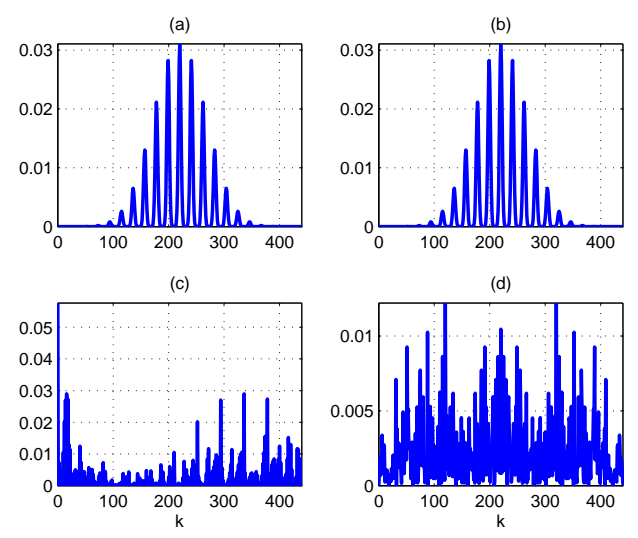

FIG. 2: (Color online). Distribution of the two-spin probability $\langle\Psi(t) \mid \Psi(t)\rangle$ as function of the number of state $k$ in the long basis $\{0, \ldots,(2 S+1)(2 S+1)\}$ for $S=10$. (a) for the initial state, $t=0$, (b) before the first phase kick at $t<t_{1}$, (c) after the first kick, but before the second kick at $t_{1}<t<t_{2}$, and (d) after the second kick $t>t_{2}$.

we are able to spread the distribution making it more uniform [18, 19], while the Hamiltonian evolution produces correlation between spin components in the angular momentum basis, leading to entanglement.

In Fig. 2, we plot the distribution of the two-spin probability $\langle\Psi(t) \mid \Psi(t)\rangle$ as a function of the state number $k$, corresponding to the basis $\left|k_{1}, 10\right\rangle\left|k_{2}, 10\right\rangle$, in the following way, $|-10,10\rangle|-10,10\rangle$ corresponds to $k=0$, the next state $|-10,10\rangle|-9,10\rangle$ corresponds to $k=$ 1, and so on. For the initial state (Fig. 2 (a)), the distribution is centered on $|0,10\rangle|0,10\rangle$, that is, $k=2 S(S+1)+1=221$. The Hamiltonian evolution does not affect the distribution of the amplitudes of the components of the basis $k\left(\left|k_{1}, S\right\rangle\left|k_{2}, S\right\rangle\right)$ as shown by Fig. 2 (b). It can be seen in Fig. 2 (c) that after the first instantaneous rotation of each spin, the distribution of $\langle\Psi(t) \mid \Psi(t)\rangle$ spreads over the states $k$, this effect is increased after the second instantaneous rotation of each spin (Fig. 2 (d)).

For semi-integer spins $(S=(2 n+1) / 2, n=0,1, \ldots)$, the situation is slightly different: the initial distribution is similar to Fig. 2 (a), but instead of having a single maximum at the center, it has two maxima, which leads to non-essential (but observable) differences in the dynamics.

The analytical expressions are very cumbersome for higher spins, so that we have to use numerical optimization for $S \geq 3 / 2$. 


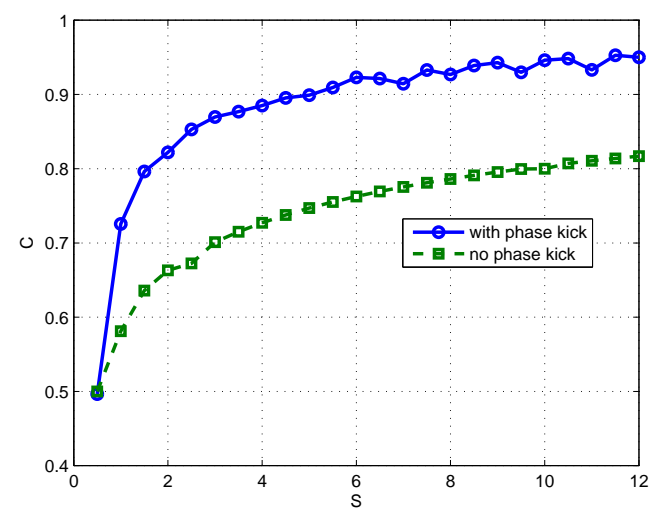

FIG. 3: (Color online) The behavior of $\left\langle\mathcal{C}_{I}\right\rangle$ against the spin number $S \leq 12$. The (green) squares joined by the dashed line correspond to the two-spin system under the Hamiltonian evolution given in Eq. 1, The (blue) circles joined by a continuous line correspond to the enhanced behavior, for times $t \geq t_{2}$.

As noted before, for the simplest case, when $S=1 / 2$, the system oscillates between the separable and maximally entangled states and the kicks produce just a phase shift, that does not affect the dynamics.

For $S=1$, we have been able to analytically optimize $\mathcal{C}_{I}$, and found that the entanglement acquires its maximum value when the kicks are applied at times $t_{1} \approx 1.6$ and $t_{2} \approx 3.9$ (which is also in accordance with the numerical results). For these times, $\mathcal{C}_{I}$ reaches the maximum $\mathcal{C}_{I}^{\max } \approx 0.98$ at times $t \approx 7.1+2 \pi n$, which should be compared with that obtained by the pure Hamiltonian evolution, where $\mathcal{C}_{I}^{\max } \approx 0.88$. However, it is more important that after the second kick the quasi-periodical behavior of $\mathcal{C}_{I}$ has the minimal value $\mathcal{C}_{I}^{\min } \approx 0.57$, which means that the spins are entangled at any time $t>t_{2}>t_{1}$, as shown in Fig. 1 (a), and $\left\langle\mathcal{C}_{I}\right\rangle$ increases from $\left\langle\mathcal{C}_{I}\right\rangle \approx 0.58$ to $\left\langle\mathcal{C}_{I}\right\rangle \approx 0.71$.

In Fig. 1 (b), we plot the entanglement dynamics for $S=10$. In this case, the effect of enhancing the maximum of entanglement for $t>t_{2}$, is not that pronounced as for $S=1$, it increases from $\mathcal{C}_{I}^{\max } \approx 0.90$ to $\mathcal{C}_{I}^{\max } \approx 0.96$. However, another important effect emerges: the transient nature of entanglement becomes significantly improved, i.e. after the second kick $\mathcal{C}_{I}$ rapidly oscillates with a very small amplitude around its average value $\left\langle\mathcal{C}_{I}\right\rangle \approx 0.95$. So that the spins are entangled for all times $t>t_{2}$, with a high value. On the other hand, we observe, that in the case of pure Hamiltonian evolution $\mathcal{C}_{I}$ periodically oscillates between its 
$\operatorname{maximum} \mathcal{C}_{I}^{\max } \approx 0.90$ and zero.

The average $\mathcal{C}_{I}$ grows slowly as $S$ increases, and even if the maximum and minimum are getting closer, the rate of increase of the average is not as fast as could be expected. This is a consequence of the growing character of $\left\langle\mathcal{C}_{\mathcal{I}}\right\rangle$ for the Hamiltonian dynamics for large $S$ as seen from Eq. (31). To represent such behavior clearly we plot in Fig. (3) $\left\langle\mathcal{C}_{I}\right\rangle$ for the Hamiltonian evolution (squares) and after applying the sequence of kicks (circles), numerically obtained.

\section{DISCUSSION}

We have studied the dynamics of a two-spin entanglement assisted by two phase kicks. The instantaneous kicks were applied at times such that the maximum of $\mathcal{C}_{I}$ has been (numerically) optimized. We found that such kicks not only lead to an enhancement of the two-spins maximum entanglement, but also to a substantial increasing of the minimum and average values of $\mathcal{C}_{I}$ for times after the second kick. Such an effect becomes much more pronounced for large spins $S \gg 1$, when $\mathcal{C}_{I}$ tends to a constant value very close to unity.

It is worth noting that the interaction Hamiltonian similar to (11) appears in several effective processes. In particular, when two non-interacting spins are embedded in a dephasing environment [6]. The Hamiltonian describing such evolution has the form $H=\Omega_{1} S_{z 1}+\Omega_{2} S_{z 2}+\sum_{k} \omega_{k} a_{k}^{\dagger} a_{k}+\left(S_{z 1}+S_{z 2}\right) \sum_{k} g_{k}\left(a_{k}+a_{k}^{\dagger}\right)$, such that $\omega_{k} \gg \Omega_{1,2}$, $g_{k}$. The fast field is effectively decoupled from the spin subsystem and thus can be adiabatically eliminated in a standard way [20] by applying a small unitary transformation $U=\exp \left[\left(S_{z 1}+S_{z 2}\right) \sum_{k} \epsilon_{k}\left(a_{k}^{\dagger}-a_{k}\right)\right], \epsilon_{k}=g_{k} / \omega_{k}$, so that the effective Hamiltonian, $H_{e f f}=U H U^{\dagger}$ (diagonal on the field variables) takes the form,

$$
\begin{aligned}
H_{e f f}= & \left(\Omega_{1}-\epsilon S_{z 1}\right) S_{z 1}+\left(\Omega_{2}-\epsilon S_{z 2}\right) S_{z 2} \\
& +\sum_{k} \omega_{k} a_{k}^{\dagger} a_{k}-2 \epsilon S_{z 1} S_{z 2}
\end{aligned}
$$

where $\epsilon=\sum_{k} g_{k} \epsilon_{k}$. It can be observed that the effect of bosonic bath in this case is reduced to an effective interaction between spins, which may give rise to a creation of spin entanglement and a quadratic phase shift that is not important for entanglement generation. 


\section{ACKNOWLEDGEMENTS}

The work of G.B is partially supported by CONACyT grant 47220. The work of I.S. was supported by CONACyT grant 74897 .

[1] S. Campbell and M. Paternostro, Phys. Rev. A 79, 032314 (2009); P. J. dosReis and S. S. Sharma, Phys. Rev. A 79, 012326 (2009); J. G. Oliveira, R. Rossi, and M. C. Nemes, Phys. Rev. A 78, 044301 (2008).

[2] L Amico, R. Fazio, A. Osterloh, and V. Vedral, Rev. Mod. Phys. 80, 517 (2008).

[3] H. Bechmann-Pasquinucci and W. Tittel, Phys. Rev. A 61, 062308 (2000); M. Bourennane, A. Karlsson, and G. Björk, ibid. 64, 012306 (2001); S. P. Walborn, D. S. Lemelle, M. P. Almeida, and P. H. SoutoRibeiro, Phys. Rev. Lett. 96, 090501 (2006).

[4] D. L. Zhou, B. Zeng, Z. Xu, and C. P. Sun, Phys. Rev. A 68, 062303 (2003); W. Hall, Qauntum Inf. Comp. 7, 174 (2007).

[5] A. R. R. Carvalho, F. Mintert, S. Palzer, and A. Buchleitner, Europ. Phys. J. D 41, 425 (2007).

[6] J. Dajka and J. Luczka, Phys. Rev. A 77, 062303 (2008).

[7] F. Benatti, A. M. Liguori, and A. Nagy, J. Math. Phys. 49, 042103 (2008).

[8] M. Yang, A. Delgado, L. Roa, and C. Saavedra, Opt. Comm. 282, 1482 (2009).

[9] A. Vaziri, J.-W. Pan, T. Jennewein, G. Weihs, and A. Zeilinger, Phys. Rev. Lett. 91, 227902 (2003).

[10] A. Bayat and V. Karimipour, Phys. Rev. A 75, 022321 (2007).

[11] N. D. Mermin and G. M. Schwarz, Found. Phys. 12, 101 (1982).

[12] J. M. Radcliffe, J. Phys. A 4, 313 (1971); F. T. Arecchi, E. Courtens, R. Gilmore, and H. Thomas, Phys. Rev. A 6, 2211 (1972).

[13] C. C. Gerry, A. Benmoussa, E. E. Hach III, and J. Albert, Phys. Rev. A 79, 022111 (2009).

[14] G. Tóth, C. Knapp, O. Gühne, and H. J. Briegel, Phys. Rev. A 79, 042334 (2009).

[15] M. Kitagawa and M. Ueda, Phys. Rev. A 47, 5138 (1993).

[16] P. Rungta, V. Bužek, C. M. Caves, M. Hillery, and G. J. Milburn, Phys. Rev. A 64, 042315 (2001); P. Rungta and C. M. Caves, ibid. 67, 012307 (2003). 
[17] D. Shindo, A.Chavez, S.M. Chumakov, and A. B. Klimov, J.Opt. B.: Quant.Sem.Opt., 634 (2004).

[18] B. Kraus, K. Hammerer, G. Giedke, and J. I. Cirac, Phys. Rev. A 67, 042314 (2003).

[19] K. Hammerer, K. Mølmer, E. S. Polzik, and J. I. Cirac, Phys. Rev. A 70, 044304 (2004).

[20] A. B. Klimov and L. L. Sánchez-Soto, Phys. Rev. A 61, 063802 (2000); A. B. Klimov, L. L. Sánchez-Soto, A. Navarro, and E. C. Yustas, J. Mod. Opt 49, 2211 (2002). 